\title{
mobileWAY - A system to reduce the feeling of temporary lonesomeness of persons with dementia and to foster inter-caregiver collaboration
}

\author{
Philipp Jordan \\ University of Hawaii / Fraunhofer \\ Portugal - AICOS \\ philippj@hawaii.edu
}

\author{
Paula Alexandra Silva \\ Portucalense University / Fraunhofer \\ Portugal - AICOS \\ palexa@gmail.com \\ Renato Oliveira \\ Portucalense University / Fraunhofer \\ Portugal - AICOS \\ Renato.oliveira@,fraunhofer.pt
}

\author{
Francisco Nunes \\ Fraunhofer Portugal - AICOS \\ xico.nunes@gmail.com
}

\begin{abstract}
Abstract - This paper presents mobileWAY (mobile Where Are You), a system that enables caregivers of persons with dementia $(P w D)$ to remotely display dynamic, customized and illustrated information on the home television of the PwD. In particular, caregivers can, through the use of a mobile device, provide information about their identity, their whereabouts and the time remaining until they return to the house of the $P w D$. When adequate, caregivers can also define an entertainment activity for the $P w D$.

The development of mobileWAY followed an iterative and incremental user-centered design approach. Semi-structured interviews with nine informal and two professional caregivers furthered a literature review and a competitive analysis of the research projects around this scope. Building upon these, a low-fidelity prototype was created and iterated twice through usability testing with seven PwD and fourteen caregivers. Results show that PwD comprehend the displayed information and caregivers effectively use and recognize the usefulness of mobileWAY.
\end{abstract}

\section{Introduction}

In 2025, every fourth European will be older than 60 years [1]. This number is immense and will continue to grow in the next decades. Even today, senescence poses a threat to the already limited capacities of medical healthcare systems and economically exhausted governments. In 2010, more than 700 million individuals worldwide (about 10\% of the total world population) had already reached the age of $60+[2]$. Age-related, long-term chronic conditions
(CC) are both psychologically burdening and financially challenging for PwD and informal caregivers. Since the number of cases is expected to double every 20 years [2], dementia, one of the most common $\mathrm{CC}$, will play a significant role in the caregiving management of the 21 st century worldwide [3]. In the context of the present socioeconomic challenge and foreseen demographical development, innovative technology may have the potential to both reduce the exploding costs of long-term caregiving on one side and increase autonomous, assisted and informal home care on the other side. The contributions of this paper further the above-described context. First, it gives an overview of previous projects and research wherein dementia-related contexts have been supported by the use of Information and Communication Technologies (ICT), i.e.: any communication device, application or service making use of technologies such as radio, television, phones tablets, computer networks, hard- and software or satellite systems. Furthermore, it presents the results of a qualitative user research consisting of eleven semistructured interviews (with nine informal and two professional caregivers of PwD) that expand our understanding of what dementia is and what caregiving for PwD involves. Also, it introduces mobileWAY, a system to support PwD and their caregivers, which in its very essence enables caregivers to remotely display custom information and entertainment on the TV in the home of the PwD. The design, evaluation and implementation process of mobileWAY as well as the results from the evaluation with users are also presented.

The first low-fidelity prototype, a series of interface sketches framed in a cardboard mockup, was tested with a small group of participants (two PwD and four caregivers) in a home environment. After the first iteration, i.e. revision and improvement of the initial 
low-fidelity prototype, the design was re-tested with a larger group of participants (five $\mathrm{PwD}$ and ten caregivers). A questionnaire was also administered to assess the subjective satisfaction of caregivers with the system in both instances. A follow-up and final iteration (mobileWAY v2) lead to the implementation of a working prototype of mobileWAY. Results show, for the PwD, partial to full comprehensibility of the information displayed on the TV and, for the caregivers, high effectiveness and efficiency in using the system, which is controllable from a mobile tablet or a smartphone. Additionally, caregivers report that the solution would likely improve their own life as well as the life of their dementia-affected person.

\section{Background - ICT-related dementia exploration}

This section presents an overview of related projects and research in the scope of this paper. In the last decade, diverse studies linked the healthcare and ICT domains to address the needs of special target audiences using ICT for practical applications (for e.g. [4] [5] [6] [7] [8] [9]). Essentially these projects focused on promotion of independence, social inclusion and support by Assistive Technology (AT). Across these projects, foci on ease of use, learnability and memorization emerge as crucial aspects for a successful adoption and interaction with the developed solution [10].

Besides understanding how technology can be used to support everyday motor tasks (such as getting dressed), researchers are studying how it can avoid feelings of loneliness and dependence as well as how it can promote social inclusion of PwD [11]. For instance, a large study identified four main areas in which ubiquitous computing can potentially support cognitively impaired persons: prevention and detection, management of everyday life, social connectedness, and identity affirmation [12].

More recently, user-led design approaches [13] as well as innovative methods centering on empathetic relationships between designers and participants have been proposed [14]. Also, the participation of the (primary) caregivers in the design and evaluation of these systems seems beneficial as previous research confirms the importance of eliciting input not only from PwD but also their caregivers as a central issue [15]. If possible, going either in the houses or care homes of PwD is a promising method when conducting qualitative research [11].

Present day, research also shows a general tendency towards more individual and customized solutions for supporting PwD inside and outside of their homes with stationary and mobile ICT [16]. Most of above- mentioned projects and studies use prototypes or adapted devices; either a stationary, touch-driven display or a mobile phone, sometimes both simultaneously. A trend to unobtrusive and noninvasive sensor technology to promote independent living and to avoid deprivation can also be detected [17]. If available, the usage of high-density visual information, such as big pictures or videos of related persons or bygone days is identified as well [5] [6]. Other examples of solutions proposed include cognitive games to decelerate the course of the disease [18], affective virtual agents (computer characters that recognize sentiment and communicate using gestures or voice output) to recognize the mood of the PwD [19], reminiscence therapy with photo and video sharing across networks [20] and music therapy to stimulate creativity [21].

\section{Research Methodology}

The research methodology consisted of four phases.

- Phase I - Qualitative interviews with caregivers;

- Phase II - Initial low-fidelity prototype design and usability evaluation with end-users;

- Phase III - Iteration of the low-fidelity prototype design and usability evaluation with end-users; and

- Phase IV - High-fidelity prototype design and implementation.

The qualitative interviews consisted of eleven semistructured interviews performed with nine informal and two professional caregivers. These interviews were recorded, fully transcribed and coded into fragments. The primary goal of the interviews was to unveil the most important PwD and caregiver needs and concerns, which then would allow for the definition of a solution. Once the most relevant use cases and functionalities were identified, a low-fidelity prototype was created and then subjected to usability testing with two PwD (TV) and four caregivers (tablet). The Wizard-of-Oz (in which a facilitator simulates the behavior of the system) and think-aloud method (that encourages the verbalization of the participants' thoughts) were used to perform the tests in the house of the PwD along with their primary caregivers, an approach that had shown positive results in [11] [15]. Mainly, the initial TV prototype did focus on mediating information about the identity (who), activity (what) and arrival (when) of the next informal caregiver coming back to the PwD's house. In order to 
assess the subjective satisfaction with the initial system as well as its perceived potential, a questionnaire with 14 questions was also administered to caregivers.

The results from the usability evaluation of the initial low-fidelity prototype enabled the iteration of the design - mobileWAY v2. The iteration was consequently subjected to usability testing with five PwD and ten caregivers using the same methods as in the previous phase. This evaluation included six additional tasks and was conducted in a local adult day care center where the PwDs spent most of their daytime guaranteeing a familiar environment, as suggested by [11]. Caregivers were again invited to answer the questionnaire used in the initial evaluation.

In conclusion, a working prototype of mobileWAY was realized and implemented on the Android mobile operating system while following its design and development best practices. The caregiver application was implemented on an Android based tablet device while the TV application for the PwD was implemented on Google TV.

\section{Phase I - Qualitative interviews with caregivers}

The delicate and problematic nature of the disease rendered it impossible to approach PwD as interview participants. Therefore, actual relatives, informal and formal caregivers of $\mathrm{PwD}$ participated as interviewees. Eleven interviews were performed either by phone or in person to nine informal and two professional caregivers, respectively totaling 213 and 80 minutes of audio recordings. Afterwards, the interviews were fully transcribed and coded into fragments (information relating to a topic of interest). These individual fragments were then categorized into groups and finally interpreted and evaluated [22].

All PwD the caregivers referred to in the interviews had Alzheimer's disease (AD) and lived by themselves at their own home or together with their relatives in an informal caregiving situation.

Besides the demographics of both PwD and caregivers, the interview script consisted of a total of fourteen questions, structured into three main areas of interest: general information, social changes and technology prerequisites. The first set with six questions assessed essential information, such as the relationship of the informal caregiver with the PwD, the age of onset, the dementia type and the early symptoms of the PwD. The second set consisted of four questions assessing the changes in the life of both $\mathrm{PwD}$ and caregiver after the early signs occurred. Also problematic and dangerous situations within the PwD's history were asked. The third set queried the usage of
AT and aids, such as memory games, calendars, post-it notes or adapted hardware in the house. Finally, the capabilities of the dementia PwD towards ICT were questioned.

After transcription and qualitative analysis, eleven categories (Table 1) emerged and enriched our understanding of the practical reality of the domain.

Table 1. Categories derived out of the interviews

\begin{tabular}{|c|c|}
\hline ESS & $\begin{array}{l}\text { Early stage symptoms (ESS) are characterized due } \\
\text { to changes in behavior, aggressiveness, memory } \\
\text { problems, changes in daily routines. }\end{array}$ \\
\hline MSS & $\begin{array}{l}\text { Middle stage symptoms (MSS) are characterized } \\
\text { by motor malfunctions as well as progressive } \\
\text { cognitive decline. }\end{array}$ \\
\hline LSS & $\begin{array}{l}\text { Late stage symptoms (LSS) are characterized by } \\
\text { the loss of basic physical abilities like walking, } \\
\text { speaking, ultimately being bedridden. }\end{array}$ \\
\hline LD & $\begin{array}{l}\text { Late diagnosis, meaning the diagnosis was later } \\
\text { than one year after the first signs occurred (onset). }\end{array}$ \\
\hline $\mathrm{CoA}$ & $\begin{array}{l}\text { Coincidence of an accident / psychological } \\
\text { problems }\end{array}$ \\
\hline Emo & $\begin{array}{l}\text { Emotional problems of the family / informal } \\
\text { caregivers of the PwD }\end{array}$ \\
\hline $\mathrm{Ch}$ & $\begin{array}{l}\text { Changes in the everyday life of the family } \\
\text { informal caregiver of the PwD }\end{array}$ \\
\hline ATP & Assistive Technology used by the PwD \\
\hline ATC & $\begin{array}{l}\text { ICT used by the family / informal caregivers of the } \\
\text { PwD }\end{array}$ \\
\hline For & $\begin{array}{l}\text { Oblivion happenings of the family / informal } \\
\text { caregivers of the PwD (forgetting medical } \\
\text { appointments or other patient-related tasks) }\end{array}$ \\
\hline Misc. & Miscellaneous, did not fit in any other category \\
\hline
\end{tabular}

The qualitative user research mostly confirmed previous findings. In short, PwD are most of the time older adults with a late dementia diagnosis and multiple other age-related diseases. Furthermore, concealment of the disease due to the cognitive decline causes a loss of hobbies and social withdrawal. The user research also indicates little to non-existent ICT experience or capacities of older adults with dementia. The TV is the only technology and device that is known and accepted in the PwD's environment while the ability to use a traditional landline phone - if existent - gets lost early. Although the ability to use landline phones vanishes over time, PwD are still able to follow a TV program (eventually being set up by a caregiver in the later stages of the disease due increased problems in handling the remote control).

Moreover, informal, multi-caregiver environments are a regular case. Families, friends and informal caregivers try, want to and do take care of their dementia-affected relative in a home setting. But they struggle psychologically and socially between keeping track of their own responsibilities in life (school, job, 
hobbies and friends) on one side, and taking care of their related or acquainted PwD on the other side.

Therefore, continuous stress and emotional difficulties in dealing with the new situation are stated in the interviews. The fear of leaving the PwD temporarily alone is one of the main causes of distress. Additionally, the interviews show that informal caregivers already organize their life around the PwD. To achieve the aforesaid, they use basic technology, such as phone calls, shared calendars and notes. The permanent integration of the caregiver in the everyday life of the PwD as well as the rare instances of overlooking disease-related tasks or medical appointments are noteworthy.

\section{Phase II - Initial low-fidelity prototype design and usability evaluation with end- users}

This section describes Phase II, in particular the design and evaluation of mobileWAY v1.

\subsection{Initial design}

The user research revealed that the TV as an individual and informative display offers limited, but unexploited, possibilities to support a PwD in cases of temporary lonesomeness. According to the caregivers' responses in Phase I, the most severe situations (for PwD and caregivers) occurred when the PwD was left alone - mostly at his or her own place - for a temporary period of time. As so, the main functionalities of the future system focus on mediating information about the identity (who), activity (what) and arrival (when) of the next caregiver coming back to the PwD's house. To address these needs, the TV module of mobileWAY utilizes the television to display dynamic, customized and illustrated information in the house of the PwD in situations of temporary caregiver absence (Figure 1).

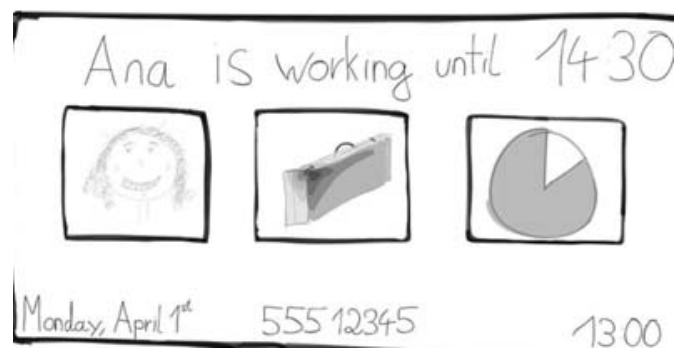

Figure 1. TV low-fidelity prototype (mobileWAY v1)

A tablet module allows the caregivers to control the information displayed on the TV regarding their whereabouts, their activity and the time until they are back at the house of the PwD (Figure 2). Organizational helps for the caregivers, even if secondary, are considered by offering multi-caregiver accessible calendars, shareable to-do lists, and a forum for information exchange. These functionalities were derived from the outcomes of Phase I, specifically from examples in which caregivers reported to interchange and forward PwD-related tasks among themselves by using notes, calendars and messages.

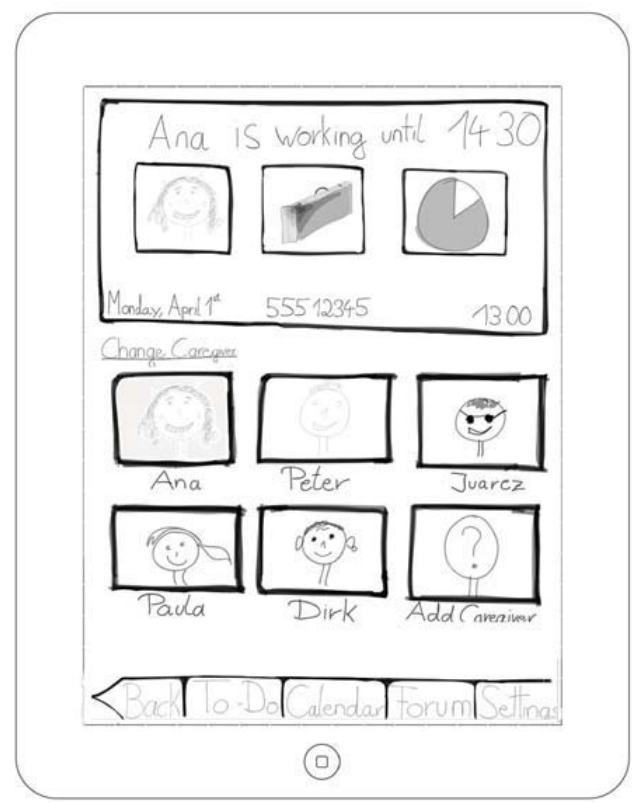

Figure 2. Tablet low-fidelity (mobileWAY v1)

\subsection{Methodology}

The initial usability tests involved two PwD and four informal caregivers to evaluate the different modules of the system. No participant had prior contact with mobileWAY to avoid skewing the results.

While the PwD evaluated the TV module, the caregivers tested the tablet module, which in turn controls the TV module. All usability tests were carried out at the home of the PwD, a familiar environment that would foster calmness and reduce their participation reluctance. In consequence, mobileWAY was tested in the ecosystem it was designed for. In fact, conducting the tests in their home (or in the day care center where they spend most of their time, see Section 6) turned out being beneficial as discussed later in the paper. This also concurs with the findings of [11].

Two facilitators were involved in each test: one guided the conversation with the participants and another simulated the interactive behavior of mobileWAY. The latter changed the UI of the paper 
prototype, according to the participant's selections, using the Wizard-of-Oz technique [22]. Furthermore, caregivers were asked to 'think aloud' [23], that is, to verbalize their thoughts throughout the interaction with the tablet and to help increase the facilitators understanding of the interaction observed. The evaluation of the TV module consisted of two stages: introduction and execution of tasks. First, the two PwD were introduced to the facilitators who explained briefly what was about to happen. After the introduction, the PwD were asked to complete six tasks (Table 2). Both PwD participants had AD, one in its initial stage and the other in the middle to late stage. Both PwD lived at their own homes together with family members serving as their informal caregivers. One participant was 87 years old while the other aged at 79 .

In detail, the PwD comprehensibility tests of the TV module assessed understanding and preference of two different time metaphors (Tasks 1-3) and three caregiver absence scenarios (Tasks 4-6). An absence scenario showed a TV screen with a different name and real photo of an informal caregiver of the PwD, the description and photo of the activity of this caregiver while being absent from the PwD and the remaining time until the return of that caregiver (displayed by both the previously chosen time metaphor and text). In each task, the facilitators asked the PwD various questions, such as: Can you tell us what you see? Do you understand the message displayed? Are you familiar with this person or place? When is your caregiver (daughter, husband) coming back?

The evaluation of the tablet module by the caregivers comprised three stages: introduction to the system, execution of three tasks, and completion of a post-test questionnaire. The average age of the four participants was $\sim 45$ and ranged from 28 to 63 years old, indicating a diverse caregiver constellation. In the introduction, the participants were briefed on the general goal of the system, the complementary system modules: TV and tablet, the privacy considerations involved, and the expected duration of the test. After the initial presentation, participants were asked to complete three tasks (Table 3).

To both ensure an early evaluation of the developed design and to capture as much input and feedback as possible, participants were invited to think-aloud [23] during their tests as well. Next, a post-test questionnaire containing 14 items was handed to the four caregivers to assess and reflect the subjective satisfaction with the system. The answer format for the questions 1 - 11 was a predetermined, 4-staged, bipolar rating scale (Table 4), while the questions 12 - 14 could be answered in an open, free format (What did you like the most?, What did you dislike the most?, What would you improve?).

\subsection{PwD Results}

PwD 1, a 79-year-old early-stage male with AD, mastered the comprehensibility tests and had no problems understanding any of the tasks (Table 2). Other than an initial association of the clock icon with a traffic sign, he was able to associate both countdown metaphors, the hourglass and clock icon (Task 1 and 2). Furthermore, he understood the concept of increase and decrease in time but was indifferent which to prefer (Task 3). Tasks 4 - 6 were fully understood, the participant was able to read the text from approximately three meters distance and responded to all questions sufficiently.

PwD 2, an 87-year-old female diagnosed for AD seven years ago. She had middle to late stage AD and a severe cognitive decline that required $24 / 7$ support. At first glance, she did not perform well (Table 2). She had severe difficulties understanding the time indicators in both cases (Task 1 - 2) and did not choose a specific icon (Task 3). Regarding the absence scenarios, she had problems recalling her relatives (daughter and granddaughter) in Task 5 and 6 and could not understand any task entirely.

Table 2. PwD comprehensibility results (v1)

\begin{tabular}{|l|c|c|}
\hline \multicolumn{1}{|c|}{ Task } & PwD 1 & PwD 2 \\
\hline $\begin{array}{l}\text { 1 - Comprehensibility test of a } \\
\text { hourglass icon }\end{array}$ & FU & NU \\
\hline $\begin{array}{l}\text { 2 - Comprehensibility test of a clock } \\
\text { icon }\end{array}$ & FU & NU \\
\hline 3 - Choose Icon & PU & NU \\
\hline $\begin{array}{l}\text { 4- Comprehensibility of caregiver } \\
\text { absence scenario 1 }\end{array}$ & FU & NU \\
\hline $\begin{array}{l}\text { 5- Comprehensibility of Caregiver } \\
\text { absence scenario 2 }\end{array}$ & FU & PU \\
\hline $\begin{array}{l}\text { 6- Comprehensibility of Caregiver } \\
\text { absence scenario 3 }\end{array}$ & FU & PU \\
$\begin{array}{l}\text { Legend: FU - Full understanding; PU - Partial } \\
\text { Understanding; NU - No understanding }\end{array}$
\end{tabular}

But partial success can also be reported. Other than forgetting the sense of the message, she had no problems in reading the text which indicates an appropriate font size of the design. More importantly, she was able to associate the caregiver with the activity (Task 4). Finally, the most satisfying results were observed in Task 5 and 6 wherein PwD 2 had neither an idea about the complete message, nor about her own daughter displayed on the TV, but was able to recall both activity images, in particular a local church and the main town hall. Here she spoke freely out of her memory and told a story about the time the town hall was constructed and the pope came for a visit and held a speech in front of a crowd. This is noteworthy, 
especially if one considers that she was unable to recognize her granddaughter neither in the low-fidelity mock-up, nor in reality when she sat next to her during the comprehensibility tests.

\subsection{Caregiver results}

All four caregivers completed the tasks successfully and efficiently. This means that without any, or very little help, the interface was understood and the tasks were performed in a straightforward way (Table 3 ).

Table 3. Usability test results (v1)

\begin{tabular}{|l|r|r|r|r|}
\hline \multirow{2}{*}{ Task } & \multirow{2}{*}{$\begin{array}{c}\text { Steps } \\
\text { needed }\end{array}$} & \multicolumn{3}{|c|}{$\begin{array}{c}\text { Steps taken across } \\
\text { participants (n=4) }\end{array}$} \\
\cline { 3 - 5 } & & Mean & Mode & \multicolumn{1}{c|}{ Max } \\
\hline 1 - Add caregiver & 8 & 8.80 & 8 & 10 \\
\hline $\begin{array}{l}\text { 2 - Change activity } \\
\text { picture }\end{array}$ & 8 & 9.30 & 8 & 12 \\
\hline $\begin{array}{l}\text { 3 - Display } \\
\text { information on TV }\end{array}$ & 12 & 13.30 & 12 & 15 \\
\hline
\end{tabular}

Table 4. Questionnaires results (v1 and v2)

\begin{tabular}{|l|c|c|c|}
\hline & \multicolumn{3}{|c|}{ Mean across participants } \\
\hline Questions & $\begin{array}{c}\mathbf{v 1} \\
\mathbf{n}=\mathbf{4}\end{array}$ & $\begin{array}{c}\mathbf{v 2} \\
\mathbf{n}=\mathbf{1 0}\end{array}$ & $\begin{array}{c}\text { Total } \\
\mathbf{n}=\mathbf{1 4}\end{array}$ \\
\hline $\begin{array}{l}\text { Q1 - mobileWAY is easy to } \\
\text { understand }\end{array}$ & 2.25 & 2.4 & 2.4 \\
\hline $\begin{array}{l}\text { Q2 - Organization and content of } \\
\text { mobileWAY is clear }\end{array}$ & 2.50 & 1.90 & 2.1 \\
\hline $\begin{array}{l}\text { Q3 - You know what to choose } \\
\text { and how to execute a task }\end{array}$ & 2.25 & 2.20 & 2.2 \\
\hline Q4 - mobileWAY is easy to use & 2.50 & 2.10 & 2.2 \\
\hline $\begin{array}{l}\text { Q5 - mobileWAY is pleasurable } \\
\text { to use }\end{array}$ & 2.75 & 2.20 & 2.4 \\
\hline $\begin{array}{l}\text { Q6 - mobileWAY will improve } \\
\text { the quality of life of the PwD }\end{array}$ & 2.25 & 1.40 & 1.6 \\
\hline $\begin{array}{l}\text { Q7 - mobileWAY will make the } \\
\text { PwD feel more secure }\end{array}$ & 2.25 & 1.20 & 1.5 \\
\hline $\begin{array}{l}\text { Q8 - mobileWAY will make the } \\
\text { PwD feel more calm }\end{array}$ & 2.25 & 1.20 & 1.5 \\
\hline $\begin{array}{l}\text { Q9 - mobileWAY will help you } \\
\text { and other caregivers to improve } \\
\text { the PwD quality of life }\end{array}$ & 2.50 & 1.50 & 1.8 \\
\hline $\begin{array}{l}\text { Q 10 - mobileWAY will help you } \\
\text { and other caregivers to manage } \\
\text { the PwD needs }\end{array}$ & 2.25 & 1.50 & 1.7 \\
\hline $\begin{array}{l}\text { Q11 - mobileWAY will make } \\
\text { you feel less concerned about the } \\
\text { PwD }\end{array}$ & 2.00 & 1.10 & 1.4 \\
\hline
\end{tabular}

Rating scale for each item: 0 - Never; 1 - Sometimes; 2 Most of the times; 3 - Always

The after-questionnaire results (Table 4, v1) indicate across all caregivers high ratings for general usability (questions $1-3$ ) and subjective satisfaction (questions 4 - 5). When asking the informal caregivers in questions $6-8$ about the benefits of the system for PwD (improved quality of life, increased PwD calmness and security feeling), one caregiver stands out negatively compared to the more positive assessments of the remaining caregivers. As mobileWAY also aims to help the informal caregivers by providing tools for organization and adjustment in multi-caregiver-environments, questions 9 - 11 were also mostly positive rated. Besides one participant, who assessed question 11 with the most negative category possible, throughout all questions and caregivers, mobileWAY was very positively assessed in terms of decreased PwD concern and increased caregiver support.

\section{Phase III - Iteration of the low-fidelity prototype design and usability evaluation with end-users}

This section describes Phase III of the methodology and details the iteration and subsequent evaluation with end-users of mobileWAY v2.

\subsection{Redesign}

The usability testing of mobileWAY v1 identified a number of design flaws. These led to mobileWAYv2, which included the revision of workflows and terminologies as well as the addition of confirmation prompts. Additionally, mobileWAY v2 also introduces new entertainment functionalities for the PwD, including features to stream music, photo slide shows and videos from a local library or global repository on the TV using the tablet as a remote control (Figure 3).

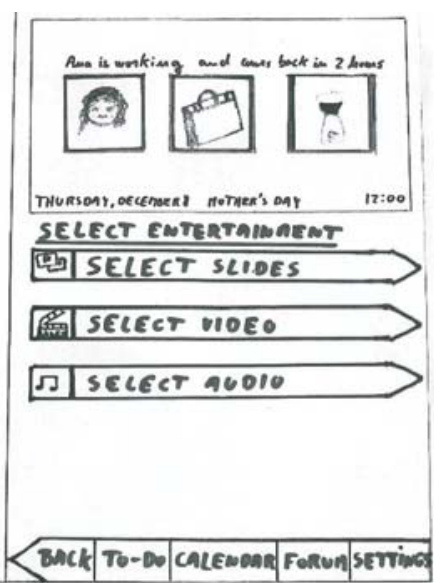

Figure 3. Low-fidelity prototype of the tablet display for caregivers with the entertainment options (mobileWAY v2) 


\subsection{Methodology}

The iterated design was tested in a similar fashion as described in Section 5.2 differing in three important aspects: a larger sample size, evaluations in a local day care center instead of the PwD home and additional test tasks. Similarly to Phase II (see Section 5), none of the participants had been exposed to the design before. Again two facilitators were present during all tests. As with the mobileWAY v1 evaluations (attendance of an informal caregiver during test), a formal caregiver was present during the evaluations in the day care center. The same as before, the formal caregivers not only created a more comfortable environment for the PwD but also fostered a better understanding of the PwD reactions and answers during the evaluations.

The TV module of mobileWAY v2 was evaluated with five PwD wherein three of the participants had early stage $\mathrm{AD}$ and the remaining two PwD had middle stage AD. The average age of all five PwD was $\sim 83$. At first, the participants were introduced to the system and the tasks to perform with the same. The first task assessed the comprehensibility of an absence scenario (Table 5, Task 1), in which a given caregiver was away performing a given activity. The second task (Table 5, Task 2) consisted of the facilitators simulating a photo slideshow on the TV module and assessing the PwD's understanding of it. The slide show consisted of various photos of their lives, their family and familiar places. The facilitators asked the PwD similar questions as in the initial evaluations to assess the comprehensibility of both tasks. As the clock icon was substantially better recognized than the hourglass icon in the initial evaluations, the time icon selection task (Table 1, Task 3) was removed.

Table 5. PwD results (v2)

\begin{tabular}{|l|c|c|c|c|c|}
\hline & \multicolumn{5}{|c|}{ PwD } \\
\hline Tasks & $\mathbf{3}$ & $\mathbf{4}$ & $\mathbf{5}$ & $\mathbf{6}$ & $\mathbf{7}$ \\
\hline $\begin{array}{l}\text { 1 - Comprehensibility of } \\
\text { caregiver absence } \\
\text { scenario }\end{array}$ & FU & FU & PU & FU & PU \\
\hline $\begin{array}{l}\text { 2- Comprehensibility of } \\
\text { the photo slide show }\end{array}$ & FU & FU & PU & FU & PU \\
\hline
\end{tabular}

Legend: FU - Full understanding; PU - Partial

Understanding; NU - No understanding

The tablet module was evaluated with a total of ten caregivers with an average age of $\sim 53$. Similar to Phase II (see Section 5), the caregiver evaluations comprised three stages: introduction and explanation of the system, execution of the tasks and post-test survey reaction (Table 4, v2). Again, the Wizard-of-Oz and think-aloud methods were utilized while - due to the redesign of the system - six new functionalities (Table
6, Tasks 4-9) were evaluated. Thus, each caregiver performed in total nine tasks when evaluating the tablet module. After the completion of all nine tasks (Table $6)$, the ten caregivers replied to the identical questionnaire (Table 4, v2) used in Phase II.

\subsection{PwD results}

PwD 3, who evaluated the prototype before the other participants, could not recognize the clock icon as a time indicator in Task 1 (Table 5). The facilitators decided to add a clock face with indicative numbers to the icon. After this solitary change of the prototype, PwD 3, 4 and 6 were able to fully comprehend both, the absence scenario (Task 1) and the photo slide show (Task 2). Furthermore, PwD 5 and 7, the two participants with middle stage $\mathrm{AD}$, could not read the text and comprehend some of the pictures in both tasks (Task 1 - 2) from the given distance the other PwD were able to. Although, reducing the distance to the TV mockup increased the readability, both participants showed only partial understanding of the displayed information. Aside from the results for both tasks, we observed a positive and encouraging reaction of the participants, particularly during the photo slideshow which was well regarded and enjoyed.

\subsection{Caregiver results}

All ten caregivers successfully completed every task in the usability evaluations for mobileWAY v2 (Table 6). Across all participants and tasks, the maximum extra number of steps a caregiver took to solve a task occurred in Task 2 with a total of three additional steps. A possible learning effect after Task 3 could be held accountable for the mostly flawless performance in Tasks 4 - 9.

Table 6. Usability test results (v2)

\begin{tabular}{|l|c|c|c|c|}
\hline \multirow{2}{*}{ Tasks } & \multirow{2}{*}{$\begin{array}{c}\text { Steps } \\
\text { needed }\end{array}$} & \multicolumn{3}{c|}{$\begin{array}{c}\text { Steps taken across } \\
\text { participants (n=10) }\end{array}$} \\
\cline { 3 - 5 } & 10 & 10.1 & 8 & 10 \\
\hline 1- Add caregiver & 9 & 9.6 & 8 & 12 \\
\hline $\begin{array}{l}\text { 2- Change activity picture } \\
\text { TV - Display information on }\end{array}$ & 14 & 14.7 & 12 & 15 \\
\hline 4- Add message & 10 & 10.0 & 10 & 10 \\
\hline 5- Select video & 6 & 6.0 & 6 & 6 \\
\hline 6- Swap video with slides & 6 & 6.0 & 6 & 6 \\
\hline $\begin{array}{l}\text { 7- Change activity } \\
\text { duration icon }\end{array}$ & 4 & 4.1 & 4 & 4 \\
\hline 8- Hide message bar & 4 & 4.1 & 4 & 5 \\
\hline 9- Hide entertainment bar & 4 & 4.0 & 4 & 4 \\
\hline
\end{tabular}


Additionally, the caregivers mentioned a few terminology imperfections and labeling suggestions.

The after-questionnaire analysis of ten participants (Table 4, v2) demonstrates an overall positive assessment of mobileWAY v2. The general usability and satisfaction using the application was positively assessed (questions 1-5). An increased quality of life for the PwD (questions 6 to 8 ) and improved caregiver organization and less burden (questions 9 to 11) were mostly answered with "Sometimes". The results for these questions differ compared to the initial assessment of mobileWAY v1.

\section{Phase IV - High-fidelity prototype design and implementation}

The previous sections described how the conceptualization, design and evaluation of the mobileWAY system evolved; these were the backdrop of the system high-fidelity design and implementation. In this phase, a graphic designer created the highfidelity prototypes of all screens and components. Simultaneously, an analysis of the current state-of-theart technologies regarding mobile platforms and tools was performed. The outcome of this analysis led to the choice of the Android mobile operating system - an open and flexible platform that is being widely adopted to implement the tablet module. The TV module was subsequently implemented on Google TV. Figure 4 illustrates the system's physical architecture, where a server, powered by Ruby on Rails, serves as a broker between the tablet device and the Google TV to transmit and update the TV information. The multicaregiver functionalities were implemented using Google's existing Web applications, such as Google Calendar and Google Tasks.

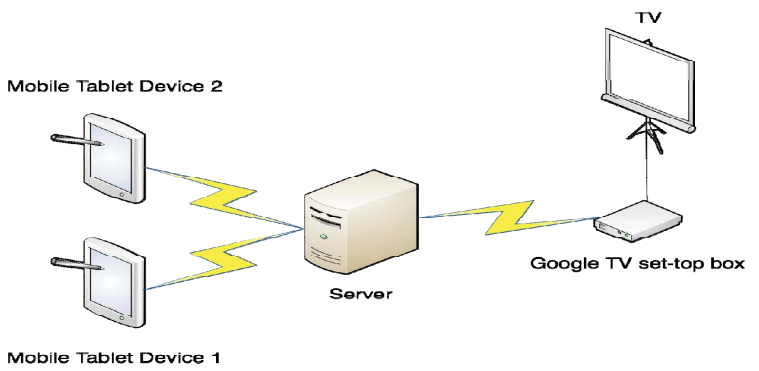

Figure 4. Physical architecture of mobileWAY

\section{Discussion}

This research used a user-centered design process with two iterations to implement a prototype system to support early-stage PwD in their home with their caregivers around. The design decision for a passive, stationary TV module for the PwD and an interactive mobile module for the caregivers originated from the theoretical and qualitative user research. As the idea of displaying content remotely on a TV screen may appear simple and obvious at first glance, the authors recall the major cognitive decline an early-stage PwD has to deal with. The balancing act between the user research outcomes, the available technological possibilities and the costs of additional hardware concluded in an easy-to-use application which informs PwD about the whereabouts and time remaining of the next caregiver who is coming back to their house. Multi-caregiver environments - which represent the reality - are addressed by specific functionalities such as shared calendars and to-do lists for the caregivers and the possibility to push notifications across multiple devices (Figure 4). Furthermore, the addition of entertainment options in Phase III, such as slideshow functionalities or music and video playback, empowers caregivers to provide an even richer caregiving experience for their dementia-affected relatives.

The results of the two evaluations performed with mobileWAY show that the PwD were fairly able to understand the information displayed on the TV module. The evaluations of the tablet module for caregivers reveal both ease of use and high usability. The questionnaires show a perceived improvement of life for both user groups (caregivers and PwD) from the caregivers' point-of-view.

These positive results regarding the evaluation and perceived impact of mobileWAY gain even greater relevance when considering that this system requires no rearrangement in the house of the $\mathrm{PwD}$, nor the usage of stigmatizing devices, such as wristbands or necklaces; these are likely to impose undesired, uncomfortable feelings on the PwD ultimately leading to behavioral changes that can affect their social life.

In addition, mobileWAY uses available, widespread and inexpensive technology, such as internet- or 3G-capable open source mobile devices. As so, the tablet module can run on any Android mobile device and is easily distributable as an app through the Google Play Store. The TV module on the other side is a non-invasive, unobtrusive device, which can run on any internet-capable TV or display situated in the home of a PwD. For these reasons, mobileWAY would be financially feasible to acquire for a wide target audience, especially in contrast to costly highend, complete assistive living environments.

Furthermore, mobileWAY empowers particularly the informal caregivers, who are likely to know the PwD for a lifetime, to use the system with appropriate photos of real places or friends and relatives to inform and maybe even stimulate their memory, as the results from PwD 2, 5 and 7 confirm. This customization with 
real photos of familiar persons and common places is one big advantage of mobileWAY and might drive the development of better and more comprehensive systems and methodologies. For instance, Kuwahara, et al. 2006 [20] used photos and video for reminiscent therapy, which is effective for increasing the selfesteem and reducing behavioral disturbances of PwD. The results of the evaluations with mobileWAY seem to indicate that this system may also offer potential in this area. One drawback of mobileWAY is the temporary or permanent attendance of the primary caregiver as an important prerequisite in this context since it is neither replacing human caregiving nor a complete round-the-clock surveillance system, ultimately leaving control and responsibility entirely on the caregivers' shoulders.

Despite the observed potential of the TV module, it is impossible to ensure whether the PwD actually pays attention to the TV. Also, the TV module is limited regarding the interaction possibilities for the $\mathrm{PwD}$. On the other hand, these constraints also minimize PwD's errors, while still offering a dynamic, customized and integrated display. The integration in the TV broadcast was out of the scope of this paper, but annoyance or agitation of the PwD in this situation may be possible.

Arguably the sample size for both groups (PwD and informal caregivers) in Phase II and III is fairly limited and evaluations with more users are desirable to further validate the results; however, the comprehensibility and usability evaluations do indicate promising results and justify further research and development. Moreover, the difficulty of accessing dementiaaffected subjects is a given fact and increasing the number of test participants is not a panacea. Given the circumstances of this area of research, the authors consider the results gathered so far to be meritorious. Both evaluation phases took place in environments which are familiar to the PwD. While the first evaluation phase took place at the houses of the PwD's, the second evaluation phase took place at the adult day care center where the PwD participants spend most of their daytime, which is somewhat of a second home for them. This decision concurs with the studies of [11] and did foster a calm, comfortable and relaxed environment for the tests, in particular for the PwD.

The PwD's familiarity with the environment, as well as the involvement of the informal caregivers in the evaluations, increased the PwD's trust, calmness and participation willingness. Since it may be odd for a PwD to face strangers asking questions, a relative with much more experience about the individual circumstances and situation of the PwD was invited to join the evaluations. The fact that a formal caregiver was present during the tests of the second evaluation phase reinforced this feeling of comfort even more.
The presence of the formal caregiver was also important to the extent that they helped facilitators obtain a better understanding of the PwD's answers.

\section{Conclusions and future work}

This paper proposes mobileWAY, a system to reduce the feeling of temporary lonesomeness of PwD and to foster inter-caregiver collaboration. This system provides two modules, one aimed at PwD, which runs on a TV, and another targeted at PwD's caregivers, which runs on a mobile device. Having been iteratively developed, this system was subjected to two usability evaluations with end-users. The evaluations show promising results not only in terms of the general comprehensibility of the system but also in terms of usability and perceived potential future interest and impact of the system.

The conceptualization of mobileWAY builds upon a review of current projects and research in this area as well as a thorough qualitative research performed with nine informal and two professional caregivers of PwD. This also constitutes a valuable contribution of this paper, which further documents and extends the knowledge of the research community in the area of dementia-related contexts.

User-centered design approaches for PwD or cognitive-impaired user groups are still in their early stages and would benefit from new or adapted design guidelines. This is also true for usability evaluation in such delicate contexts. For this project, a simple, but effective way of assessing the PwD understanding of the TV module was used and led to the involvement of the caregivers in the tests. Further, the tests were performed either at the home of the PwD or at the adult day care center where they spend most of their day time; this fostered an environment of trust, calmness and participation willingness.

As the system is currently implemented, it is important to ensure that more usability tests and longterm trials are carried out with PwD and caregivers. Adapting and optimizing of mobileWAY for this target audience will continue to be one of the system's central aspects. This process should benefit from an active and systematic discussion with clinicians and caregivers. In order to better address these aspects, future evaluations should take account of the Mini-Mental State Examination scores (MMSE) of the participating PwD. Also, the appropriate message duration or the adequate fonts and photo dimensions of the TV module should be researched, evaluated and addressed in future tests and versions of the system.

Another point that could be further investigated relates to the integration of mobileWAY into the 
regular TV broadcast, for example by the introduction of notifications in situations where the PwD enjoys a movie. The integration in personalized health information management systems is one of many longterm ideas for this project. The addition of other modalities is another facet that could be studied, such as the acoustic modality as an auditory reminder. For example, someone might imagine a predetermined, reoccurring voice output of the text message displayed on the TV. By adding this simple feature, a visual and acoustic representation of the information can be easily realized. Sensory feedback for the caregiver devices would also add value to the system. Moreover, in the future a smartphone version of mobileWAY should be designed and implemented. Herein, a redesign of the existing design to attribute the significantly smaller display sizes will be necessary.

Finally, while caregivers can currently display entertainment in the form of slides, audio or video to the PwD, it could be interesting to expand on them and provide other new possibilities, for instance, latest news from friends and family. While these options present benefits as PwD are entertained, it is also important to ensure that additional entertainment options do not suffer from information overload, considering the target audience of this research.

\section{References}

[1] ENABLE, "ENABLE Newsletter (volume 1, issue 1)," December 2007. [Online]. Available: http:// tinyurl.com/6k2yvvj. [Accessed July 2011].

[2] International Alzheimer's Disease, "World Alzheimer Report,” 2009. [Online]. Available: http://tinyurl.com/77ppv9k. [Accessed July 2011].

[3] World Health Organization, "Innovative Care for Chronic Conditions: Building Blocks for Action,” 2002. [Online]. Available: http://tinyurl.com/ 6b6b9bo. [Accessed July 2011].

[4] N. Alm, G. Gowans e A. Astell, "Circa: computer interactive reminiscence and conversation aid," 2001. [Online]. Available: http://tinyurl.com/cye5s. [Accessed July 2011].

[5] Cogknow consortium, "Cogknow - Helping people with mild dementia to navigate their day," 2009. [Online]. Available: http://tinyurl.com/ 6xxg3do. [Accessed July 2011].

[6] Cogknow, "About Us," 2010. [Online]. Available: http://tinyurl.com/3byeodn. [Accessed July 2011].

[7] Netcarity, "Netcarity Fact Sheet," 2011. [Online]. Available: http://tinyurl.com/3tjttd4. [Accessed July 2011].

[8] Senior, "Project Description," 2008. [Online]. Available: http://seniorproject.eu/. [Accessed July 2011].

[9] M. N. K. Boulos e eCAALYX Project Consortium, “An Enhanced Ambient Assisted Living Experiment for Older
People with Multiple Chronic Conditions," em 2nd International Symposium on Applied Sciences in Biomedical and Communication Technologies, Bratislava, 2009.

[10] R. Hubert, "Accessibility and usability guidelines for mobile devices in home health monitoring," SIGACCESS Accessible Computing, vol. 84, p. 26-29, 2006.

[11] J. P. Wherton e A. F. Monk, "Technological opportunities for supporting people with dementia who are living at home," International Journal of Human-Computer Studies, vol. 66, pp. 571 - 586, 2008.

[12] M. Morris e J. Lundell, “Ubiquitous Computing for Cognitive Decline: Findings from Intel's Proactive Health Research," Intel Corporation, 2003.

[13] R. Orpwood, J. Chadd, D. Howcroft, A. Sixsmith, J. Torrington, G. Gibson e G. Chalfont, "Designing technology to improve quality of life for people with dementia: user-led approaches," Universal Access in the Information Society, vol. 9, n. ${ }^{\circ}$ 3, pp. 249-259, 2010.

[14] S. Lindsay, K. Brittain, D. Jackson, C. Ladha, K. Ladha e P. Olivier, "Empathy, participatory design and people with dementia," 2012.

[15] A. Astell, N. Alm, G. Gowans, M. Ellis, R. Dye e P. Vaughan, "Involving older people with dementia and their carers in designing computer based support systems: some methodological considerations," Universal Access in the Information Society, vol. 8, n. ${ }^{\circ}$ 1, pp. 49-58, 2009.

[16] M. Mulvenna, S. Martin, S. SÃåvenstedt, J. Bengtsson, F. Meiland, R. M. DrÃ es, M. Hettinga, F. Moelaert e D. Craig, "Designing \& evaluating a cognitive prosthetic for people with mild dementia," 2010.

[17] J. Biswas, K. Sim, W. Huang, A. Tolstikov, A. Aung, M. Jayachandran, V. Foo e P. Yap, "Sensor based micro context for mild dementia assistance," 2010.

[18] F. Makedon, R. Zhang, G. Alexandrakis, C. B. Owen, H. Huang e A. J. Saykin, "An Interactive User Interface System for Alzheimer's Intervention," em Proceedings of the 3rd International Conference on PErvasive Technologies Related to Assistive Environments, New York, 2010

[19] Y. Sakai, Y. Nonaka, K. Yasuda e Y. I. Nakano, "Listener agent for elderly people with dementia," 2012.

[20] N. Kuwahara, S. Abe, K. Yasuda e K. Kuwabara, "Networked reminiscence therapy for individuals with dementia by using photo and video sharing," 2006.

[21] P. Riley, "How can technology support musical creativity for people with dementia?," 2007.

[22] J. Lazar, J. H. Feng e H. Hochheiser, Research methods in human-computer interaction, United Kingdom: John Wiley \& Sons Ltd, 2010.

[23] J. Nielsen, Usability Engineering, San Diego: Morgan Kaufmann, 1993. 